\title{
Formation and superconducting properties of predicted ternary hydride ScYH6 under pressures
}

\author{
Yongkai Wei ${ }^{1}$, Lei Kang ${ }^{2}$, Jiaonan Yuan ${ }^{1}$, guangfu ji ${ }^{3}$, and Dong-qing $\mathrm{Wei}^{4}$ \\ ${ }^{1}$ Henan University of Technology \\ ${ }^{2}$ Guangdong Pharmaceutical University \\ ${ }^{3}$ Institute of Fluid Physics, Chinese Academy of Engineering Physics \\ ${ }^{4}$ Shanghai Jiao Tong University
}

June 15, 2020

\begin{abstract}
Ternary metal hydrides play an essential role in the search for conventional high-temperature superconductors because they can be synthesized under mild condition and recovered at ambient pressure. It has been widely accepted that the electronic structure, metallization pressure and superconducting behavior of binary hydrides can be adjusted effectively by doping, replacing or introducing a new element. In this work, yttrium hydrides were chosen as parent hydrides while scandium was considered as the doped element to perform systematical crystal structure searches on the Sc-Y-H system under pressure. A new ternary hydride ScYH6 was found according to PSO calculations, and it presents high symmetric character below 150 GPa with a Pm-3 structure (cP8), then a P4/mmm phase (tP8) becomes favorable from $150 \mathrm{GPa}$. Importantly, cP8-ScYH6 is dynamically stable under pressure as low as $0.01 \mathrm{GPa}$ with a $\mathrm{Tc}$ of $32.110 \mathrm{~K}$ for Coulomb pseudopotential $\mu^{*}=0.13$, indicating ternary hydrides are promising candidates in the search for superconductors which can be synthesized under mild conditions in hydrogenrich materials. The analysis through "triangle straight-line method" (TSLM) compared with enthalpy difference calculations showed the most reasonable synthesis pathway of ScYH6 is in the whole studied pressure range. The Tc of ScYH6 takes a linear relationship with pressure up to $52.907 \mathrm{~K}$ under $200 \mathrm{GPa}$. The lattice dynamical calculations demonstrate the $\mathrm{H}$ atoms in both cP8 and tP8 structures make crucial contributions to the superconducting behavior of ScYH6. These findings can further reveal the influence of doping, replacing and introducing new element on superconducting behavior of binary hydrides.
\end{abstract}

\section{Hosted file}

manuscript.doc available at https://authorea.com/users/333384/articles/459604-formation-andsuperconducting-properties-of-predicted-ternary-hydride-scyh6-under-pressures 